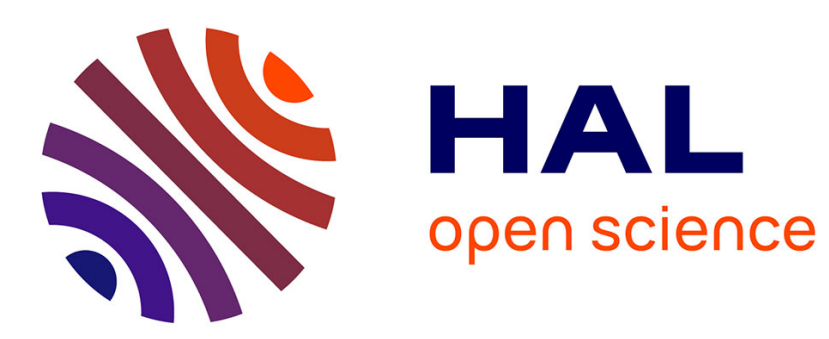

\title{
A Flexible Physical Layer for LPWA Applications: Simulations and Field Trials
}

\author{
Valérian Mannoni, Vincent Berg, François Dehmas
}

\section{To cite this version:}

Valérian Mannoni, Vincent Berg, François Dehmas. A Flexible Physical Layer for LPWA Applications: Simulations and Field Trials. The IEEE World Forum on Internet of Things (WF-IoT) 2019, Apr 2019, Limerick, Ireland. cea-02183541

\section{HAL Id: cea-02183541 https://hal-cea.archives-ouvertes.fr/cea-02183541}

Submitted on 15 Jul 2019

HAL is a multi-disciplinary open access archive for the deposit and dissemination of scientific research documents, whether they are published or not. The documents may come from teaching and research institutions in France or abroad, or from public or private research centers.
L'archive ouverte pluridisciplinaire HAL, est destinée au dépôt et à la diffusion de documents scientifiques de niveau recherche, publiés ou non, émanant des établissements d'enseignement et de recherche français ou étrangers, des laboratoires publics ou privés. 


\title{
A Flexible Physical Layer for LPWA Applications: Simulations and Field Trials
}

\author{
Valérian Mannoni, Vincent Berg and François Dehmas \\ CEA, LETI, Minatec Campus, 17 rue des Martyrs, 38054 Grenoble, France \\ \{valerian.mannoni, vincent.berg, francois.dehmas\}@ cea.fr
}

\begin{abstract}
Future Low Power Wide Area (LPWA) systems are expected to provide support for a wider range of applications: faster throughput and performance for lower levels of latency is hence forecast for a similar battery lifetime. These contradictory requirements lead to consider a flexible physical layer operating for different modes from low data rate, low power consumption, long range to high data rate. This new flexible physical layer approach is presented in this paper: simulation performance is given and compared to field trial measurements using a software defined radio implementation. Finally, field measurement comparison to state of the art LoRa is performed. The paper demonstrates with these measurements some practical benefits of the new approach.
\end{abstract}

Index Terms-LPWA, Flexible physical layer, Field Trials, Multicarrier, Turbo processing

\section{INTRODUCTION}

The installed base of the Internet of Things (IoT) devices is forecast to grow to almost 31 billion worldwide by 2020 . Low power wide-area networks (LPWA) are expected to make a significant part of the almost 2.6 billion Machine-to-Machine (M2M) connections that will be in place by that time [1]. LPWA is a generic term for a group of technologies that enable wide area communications at low cost and long battery life (Sigfox, LoRa, RPMA, NB-IoT, Weightless-P, IEEE 802.11ah) [2]. Among them, LoRa and Narrow Band-IoT (NB-IoT) are the most prominent LPWA technologies [1].

On the one hand, LoRa operates in a non-licensed band below $1 \mathrm{GHz}$ and relies on a proprietary spread spectrum modulation scheme that is derived from chirp spread spectrum modulation (CSS) to provide long range operation. CSS provides coverage by trading data rate for sensitivity. NBIoT, on the other hand, is a standardized solution that has been defined in Release 13 of the 3GPP specification [3]. It considers both single-tone transmission and Single Carrier Frequency Division Multiplexing (SC-FDM) to limit peak-toaverage power ratio (PAPR). Although sometimes regarded as a new air interface, its physical layer is a low power long range derivation of LTE [3]. Many features of LTE, including handover, measurements to monitor the channel quality, carrier aggregation, and dual connectivity have been removed to reduce device costs and minimize battery consumption.

These first generations of LPWA systems have brought coverage for long battery duration, future generations are expected to provide faster data rates and/or lower latency for similar battery lifetime to extend the range of applications the technology can deliver. The requirements evolution of LPWA connectivity have led to reconsider the physical layer for these

978-1-5386-4980-0/19/\$31.00 @2019 IEEE

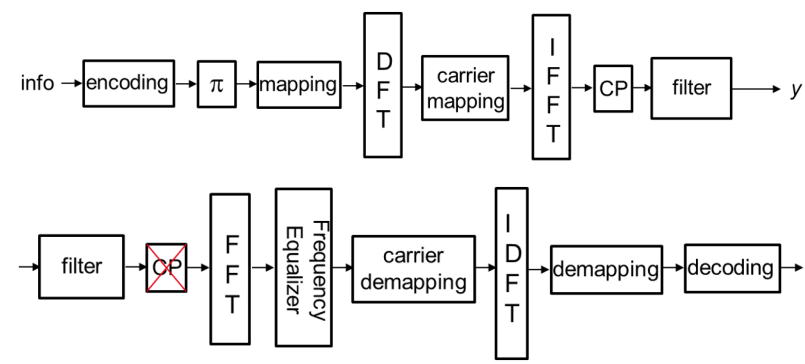

Fig. 1. Block diagram for a flexible physical layer transmitter(top)/receiver(bottom) adapted to LPWA system.

types of systems. A new flexible approach for LPWA imposed by the contrary requirements of long-range, low power consumption and higher throughput has been introduced in [4]. The authors of [4] concluded that the most adapted waveform for LPWA operation is highly dependent on the propagation scenario and the connectivity application. They proposed a flexible waveform based on frequency domain processing. When data throughput is preferred, Orthogonal Frequency Division Multiplexing (OFDM) should be considered. When range and energy efficiency should be guaranteed, TurboFrequency Shift Keying (Turbo-FSK) is more appropriate. Turbo-FSK is a new waveform introduced in [5] and [6] that meets performance close to the Shannon limit for low spectral efficiency. Finally, when power consumption is of most importance but without compromise on data throughput SC-FDM should be considered. Hence, the architecture of a new flexible physical layer has been proposed and simulated [4]. Building on this new approach, the authors of this paper have implemented the new approach on a Software Defined Radio (SDR) platform and performed field trials of the new physical layer to confront the performance of the designed physical layer with an example of real world propagation environment. This paper summarizes these new results.

The paper is structured as follows: in section II, the new LPWA physical layer approach is introduced and performance in terms of range and power consumption is estimated. Section III presents the field trial measurements that have been realized: the SDR hardware platform and the hardware environment of the trials is first presented, then measurement results are given. Section IV concludes the paper.

\section{A NEW PHYSICAL LAYER FOR LPWA APPLICATIONS}

\section{A. Signal processing architecture}

Realizing that a single waveform is not adapted to the multiple scenarios that should be supported in future LPWA 
systems, the authors of [4] have selected three different physical waveforms: OFDM, SC-FDM and Turbo-FSK and demonstrated that future LPWA system should associate the three waveforms in a flexible way. By dividing a frequency selective fading channel into a number of narrow-band flat fading subchannels, OFDM can easily compensate the channel effects using a simple one-tap frequency domain equalizer. However, the main drawback of OFDM is its high PAPR. Waveforms with high PAPR values increase the linearity requirements imposed on the power amplifier and are therefore less power efficient. Single Carrier Frequency Division Multiplexing (SCFDM) adds frequency spreading to reduce the PAPR level of OFDM. It combines the benefits of a simple equalization process as performed for OFDM with a lower PAPR. Finally, Turbo-FSK is a new waveform that meets performance close to the Shannon limit for the lower spectral efficiency and so for the lower throughput. It is a constant envelope modulation, and therefore has a PAPR equal to $0 \mathrm{~dB}$. Turbo-FSK combines a $M$-ary orthogonal modulation (FSK) with a $L$ ary linear modulation (PSK) and a parallel concatenation of $\lambda$ convolutional codes enabling at the receiver a turbo decoding.

The signal processing flow of the three waveforms employs significant amount of common processing elements such as FFT/IFFT, frequency equalization, coder/decoder. The block diagram of this new physical layer is shown in Fig. 1. Its transmitter is composed of FEC encoding, interleaving and constellation mapping. A precoding DFT is solely used for SC-FDM and bypassed by the other modes. It is followed by a carrier mapping, IFFT, the insertion of a cyclic prefix and transmit filtering. The architecture overview of the receiver (Fig. 1) follows the reverse structure of the transmitter. IDFT is only applied for SC-FDM. Thus, with a specific parameterization we can address different modes from low data rate with low power consumption to high data rate. Although the complexity introduced by the multiple modes is not analyzed, the flexibility introduced by the approach should not lead to significant complexity overhead: hardware complexity of a physical layer is often dominated by the complexity of modules such as FFT and IFFT that can be highly optimized [7].

\section{B. Simulated Performance}

The performance of the different modes is then studied under frequency selective channels in terms of range and power consumption. Transmission range (Fig. 2) has been evaluated through the 3GPP Extended Typical Urban (ETU) channel and using the Open rural Hata model [8]. The following parameters from 3GPP have been considered:

- Tone spacing $\Delta f=15 \mathrm{kHz}$

- $N_{F F T}=128$, cyclic Prefix of size $N_{C P}=9$ (4.7us).

- Packet size: 1008 bits of information

- Perfect synchronization and channel state estimation

- Carrier frequency of $868 \mathrm{MHz}$

Simulations have been performed with bandwidths ranging from $45 \mathrm{kHz}$ to $1 \mathrm{MHz}$ and with a spectral efficiency from $5.8 \cdot 10^{-3}$ to 0.66 by playing on the Modulation Coding Scheme (MCS). When the transmit power is set to $14 \mathrm{dBm}$ (Maximum power allowed in the $868 \mathrm{MHz}$ ISM band), OFDM gives the

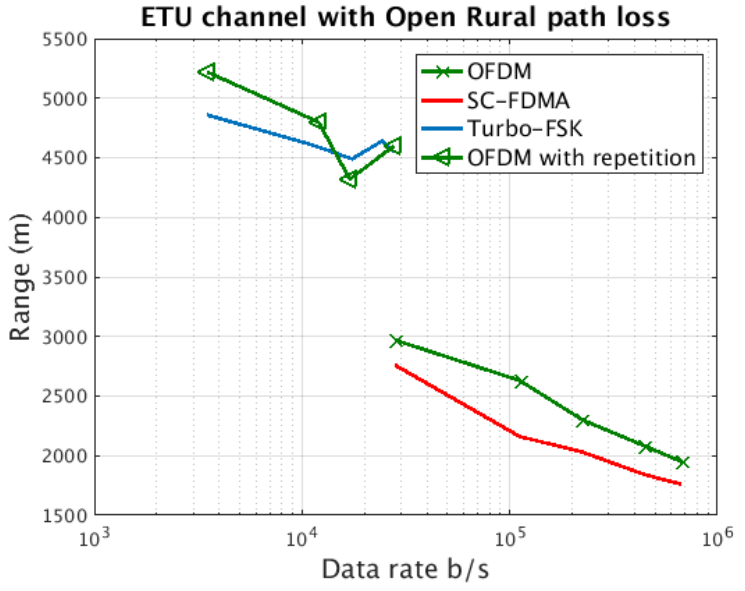

Fig. 2. Range as the function of throughput for communications through ETU channel for the different modes, OFDM, SC-FDMA and Turbo-FSK. The transmit power is set to $14 \mathrm{dBm}$.

best performance in terms of range for any given data rates (Fig. 2). It should be noted that for low bit rates (less than $30 \mathrm{~kb} / \mathrm{s}$ ) and ranges between $4.5 \mathrm{~km}$ and $5 \mathrm{~km}$, OFDM and Turbo-FSK provide similar levels of performance.

Minimizing energy consumption to increase battery lifetime is however a very important design consideration of LPWA communication systems. Therefore the impact of the physical layer on the power consumption has also been investigated. The power consumption at the transmitter is considered as the dominant effect, notably the power consumption necessary to operate the power amplifier (PA) [9]. The energy consumption per information bit, $E_{a}$, can be approximated by [10]:

$$
E_{a}=\frac{E}{N}=\frac{1}{N} \cdot\left(\frac{\epsilon}{\gamma} P_{t} T_{o n}+P_{c} T_{o n}+E_{t r}\right)
$$

where $E$ is the energy required to send a bloc of $N$ bits, $T_{o n}$, the transmission duration, $\epsilon$, the PAPR, $\gamma$ a term of power efficiency of the transmitter, $P_{t}$, the transmit power level, $P_{c}$ the power required for the internal circuit and $E_{t r}$ the energy required during transient operation. Energy consumption per transmitted bit is thus dependent on the burst duration (and hence the data rate) and the PAPR. (1) has been used to evaluate the energy consumption per transmitted information bit as a function of the throughput: i.e. for a transmission range of $6 \mathrm{~km}, P_{t}$ is adapted to reach the targeted range for the various modes of transmission. Results are summarized in Fig. 3: when energy consumption is taken into account, OFDM is the least attractive mode of operation and thanks to its null PAPR Turbo-FSK presents the best energy efficiency.

\section{Field TRIALS}

\section{A. Field Trial Set-up}

In order to validate the simulation results of Section II in a real propagation environment, field trials have been considered. The new LPWA physical layer has therefore been implemented on a hardware RF and digital Commercial OffThe-Shelves (COTS) platform in order to perform over-theair measurements. The platform is based around a Xilinx Zynq 7045: a relatively powerful FPGA/microprocessor SoC based on a dual Cortex-ARM A9 and a Xilinx Kintex FPGA. 


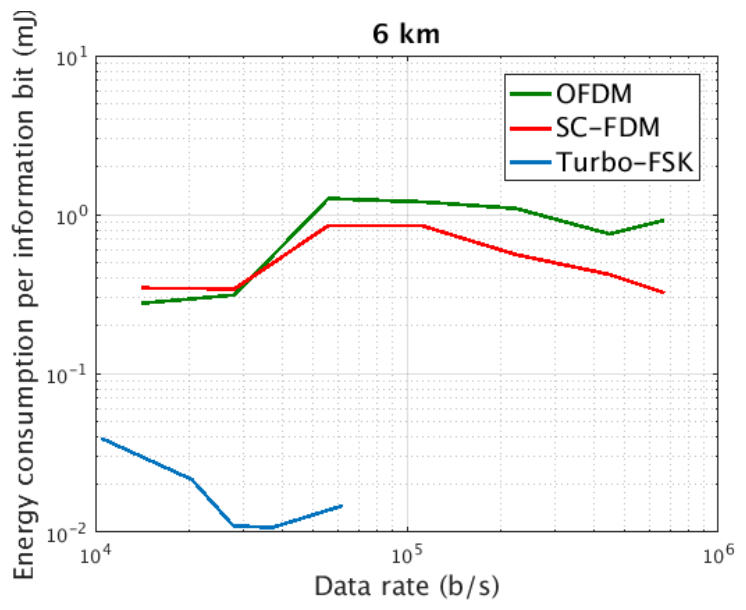

Fig. 3. Energy consumption per transmitted information bit as a function of the throughput. Targeted range of $6 \mathrm{~km}$ and packet error rate of $10^{-2}$.

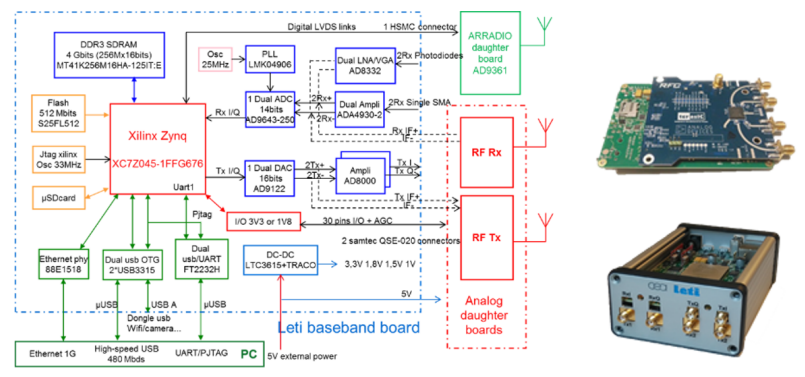

Fig. 4. Architecture of the SDR hardware platform used during the trials.

The Xilinx Zynq motherboard is completed by a RF COTS daughter-board based on analog devices AD9361 (Fig. 4). The SDR platform is interfaced, either via either a USB link or through an Ethernet link, to a Personal Computer (PC) where the physical layer, transmitter and receiver, is implemented in software (via proprietary development under Matlab ${ }^{\mathrm{TM}}$ ).

The transceivers have been deployed on the one hand on the rooftop of one building of CEA campus and on the other hand within a vehicle that can provide measurements at different locations. The transmitter deployed at top of the building rooftop is encased inside a waterproof case and represents an IoT access point (Fig 5). All measurements were carried out in downlink mode (from access point to mobile terminal), but results are also valid for uplink transmissions because the data link is assumed symmetric. The omnidirectional antenna located at the rooftop is evaluated to be located at around $20 \mathrm{~m}$ of height. However, it should be noted that this is not the highest point of the site and building situated close to the antenna could have caused some shadowing to the transmitted signal. Transmitted power has been adjusted so that the power at the output of the antenna is equal to $14 \mathrm{dBm}$ Effective Isotropic Radiated Power (EIRP).

On the mobile side, the receiver is placed inside a vehicle for mobile measurements. The receiver antenna is placed on the top of the vehicle at an approximate height of 2 meters. RF feed from the antenna is brought inside the vehicle to a Low Noise Amplifier (LNA) and finally the receiver. The mobile unit is powered by battery packs (Fig. 6).

In order to facilitate field trial measurements a Global Positioning System (GPS) receiver completes the setup. This
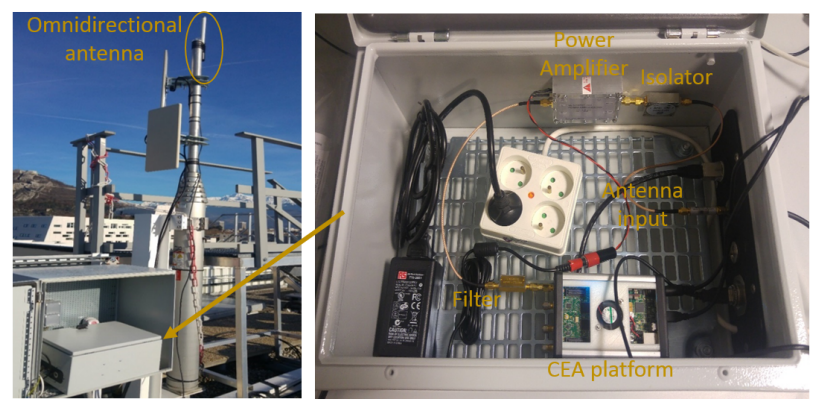

Fig. 5. Set-up of the transmitter antenna located at CEA premises and used for the field tests.

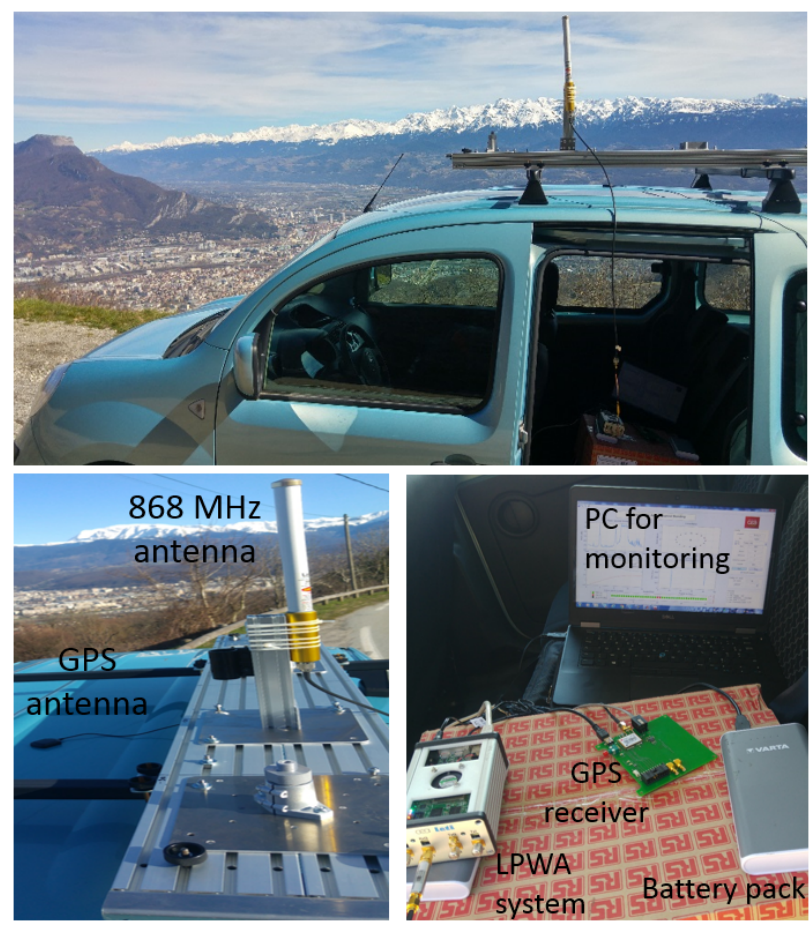

Fig. 6. Set-up of the receiver hardware and associated PC within the Field test Vehicle.

allows to evaluate when and where signal reception has been achieved or lost at the receiver.

Fig. 7 gives a typical test path that has been followed to evaluate the performance of the new physical layer for these field trial measurements. The evaluated propagation environment is considered as suburban around the access point antenna followed by a urban environment when the mobile unit is within the center of Grenoble, the city where the field trial was carried out. Then it is suburban and finally open rural. Open-rural, suburban and urban environments are mainly characterized by the rate of path loss of the transmitted signal as a function of the propagation range. When in Open rural, transmission propagation conditions are more favorable (loss is less severe). As density of building is increased, propagation conditions are more severe: path loss is increased (for the same range) and multipath is more dense causing fast variation in the channel spectrum. An example of measurements made during our field tests is shown in Fig. 7 for Turbo-FSK waveform (4 FSK / 1PSK / $\lambda=4$ ). The green dots on the figure mean that the receiver demodulates packets with a PER level of less than $10^{-2}$ (and therefore the opposite for red dots). 


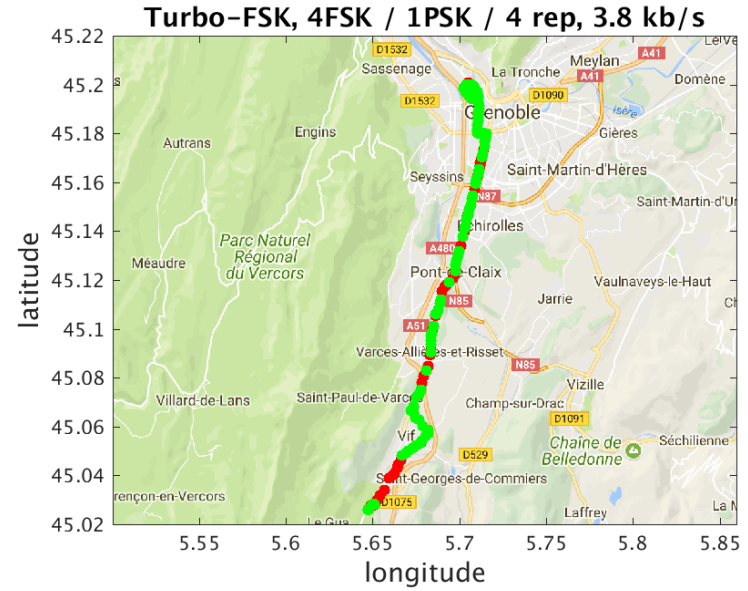

Fig. 7. Example of field trial result for Turbo-FSK 4FSK / 1PSK / $\lambda=4$.

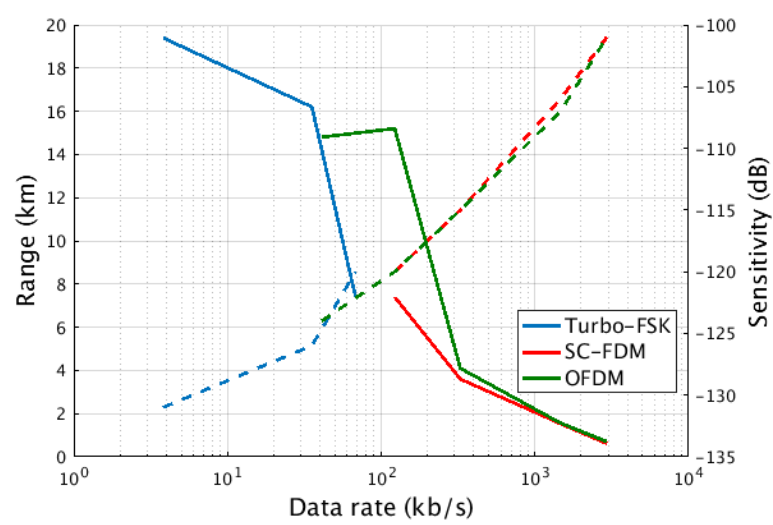

Fig. 8. Performance range (full lines) and lab sensitivity (dashed lines) as the function of data rate.

\section{B. Fied trial results}

The results obtained during the field trial of the new flexible physical layer are given in Table I and Fig. 8. The performances are given for each modes of the new flexible physical layer i.e. Turbo-FSK, SC-FDM and OFDM for different MCS and number of subcarriers $N_{u}$ for OFDM and SC-FDM. The range is the maximum distance between the base station and the device beyond for which communication can be established with a sufficiently low packet error rate (approx. $10^{-2}$ ).

Sensitivity in presence of Additive White Gaussian Noise for the various modes of operation of the new physical layer is also measured in the laboratory and given in Table I and Fig. 8. For most measurement points, a better range is provided when sensitivity is lower. This tends to suggest that range is dominated by path loss effects instead of channel conditions. Overall trends of performance seem to correlate with simulated results: Turbo-FSK is most adapted to longer ranges of operation (with a lower power consumption), while OFDM provides performance for higher data rates. SC-FDM is a fair compromise when high data rate is considered and channel conditions are not too severe.

\section{Comparison to LoRa physical Layer}

The performance between LoRa and the new LPWA physical layer, in particular when in Turbo-FSK mode has also been compared in field trials. Turbo-FSK has been chosen
TABLE I

SYNTHESIS OF THE FIELD TRIAL RESULTS

\begin{tabular}{|c|c|c|c|c|c|}
\hline Waveform & MCS & $\begin{array}{c}\text { Band- } \\
\text { width } \\
(\mathrm{kHz})\end{array}$ & $\begin{array}{c}\text { Through- } \\
\text { put } \\
(\mathrm{kb} / \mathrm{s})\end{array}$ & $\begin{array}{c}\text { Sensi- } \\
\text { tivity } \\
(\mathrm{dBm})\end{array}$ & Range \\
$(\mathrm{km})$
\end{tabular}

for its two common features with the LoRa communication system, which include low spectral efficiency and the use of a constant envelope waveform to control the transmitter's energy consumption. Similar bandwidth and data rates have been selected to perform the comparison. Results are given in terms of laboratory sensitivity and propagation range in the described set-up in Table II and Fig. 9. Let it be reminded that the physical layer of LoRa is defined by its spreading factor $S F$ and its coding rate $R_{c}$ (for a given bandwidth). In order to obtain as fair comparison as possible, the performance of both systems has been simultaneously evaluated using the same antenna for both transmission and reception via a power splitter. A first step consisted to calibrate the transmission power for both communication systems at around $12.9 \mathrm{dBm}$ which was the maximum transmission power provided by the LoRa evaluation kit that has been used. Both TurboFSK and LoRa signals are then combined using a passive combiner. Similarly at the receiver, a power splitter divides the signal coming from the LNA to both receivers (TurboFSK and LoRa). Combiner/splitter used in the communication chain introduced a loss of $3 \mathrm{~dB}$. Threfore, the performance obtained during our trials corresponds to a transmission power of $6.9 \mathrm{dBm}$ EIRP ( $3 \mathrm{~dB}$ at the transmitter and $3 \mathrm{~dB}$ at the 
TABLE II

TURBO-FSK/LORA PERFORMANCE COMPARISON DURING THE FIELD TRIAL

\begin{tabular}{|c|c|c|c|c|c|}
\hline Waveform & MCS & $\begin{array}{c}\text { Band- } \\
\text { width } \\
(\mathrm{kHz})\end{array}$ & $\begin{array}{c}\text { Through- } \\
\text { put } \\
(\mathrm{kb} / \mathrm{s})\end{array}$ & $\begin{array}{c}\text { Sensi- } \\
\text { tivity } \\
(\mathrm{dBm})\end{array}$ & $\begin{array}{c}\text { Range } \\
(\mathrm{km})\end{array}$ \\
\hline \hline LoRa & $\begin{array}{c}\mathrm{SF}=7 \\
R_{c}=4 / 5\end{array}$ & 125 & 5.8 & -123 & 1.1 \\
\hline Turbo-FSK & $\begin{array}{c}8 \mathrm{FSK}, \\
1 \mathrm{PSK}, \\
\lambda=4\end{array}$ & 131 & 7.6 & -129 & 6.8 \\
\hline \hline LoRa & $\begin{array}{c}\mathrm{SF}=7, \\
R_{c}=4 / 5\end{array}$ & 250 & 11.5 & -120 & 0.95 \\
\hline Turbo-FSK & $\begin{array}{c}16 \mathrm{FSK}, \\
1 \mathrm{PSK}, \\
\lambda=4\end{array}$ & 262.5 & 11.4 & -128 & 6 \\
\hline \hline LoRa & $\begin{array}{c}\mathrm{SF}=7, \\
R_{c}=4 / 6\end{array}$ & 500 & 19.1 & -117 & 0.8 \\
\hline Turbo-FSK & $\begin{array}{c}32 \mathrm{FSK}, \\
1 \mathrm{PSK}, \\
\lambda=3\end{array}$ & 525 & 20.3 & -126 & 5 \\
\hline
\end{tabular}

receiver).

The measured performance results are given in Table II and Fig. 9. Turbo-FSK significantly outperforms LoRa for every measured configuration. Indeed, the maximum performance range of Lora is equal to around $1 \mathrm{~km}$ whereas with the Turbo-FSK waveform ranges between $5 \mathrm{~km}$ to $7 \mathrm{~km}$ could be reached. This significant gap between the two communication systems is essentially explained by the difference in terms of channel coding (FEC). LoRa relies on a Hamming code with a coding rate between $\frac{1}{2}$ and 1 (no coding) while TurboFSK has a channel coding based on a Turbo-code scheme (associated with an iterative Max-Log-MAP decoder). Turbocodes significantly outperform Hamming code with a gap of 5 $\mathrm{dB}$ minimum (according to the coding rate) in terms of SNR [11]. This can explain the difference in coverage between both systems.

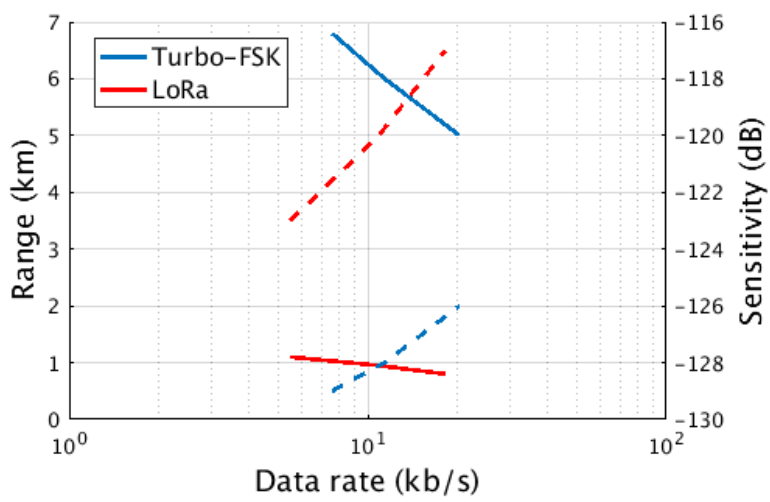

Fig. 9. Turbo-FSK / LoRa comparison: Performance range (full lines) and lab sensitivity (dashed lines) as the function of data rate.

\section{CONCLUSION}

The first generation of LPWA systems have brought coverage for long battery life. Future generations are expected to provide faster data rates to broaden the range of applications LPWA systems can provide. These new requirements for LPWA applications have led to reconsider the physical layer for these types of systems. A new flexible physical layer has hence been proposed and described in this paper. Simulation results have been used to justify the gains that are provided by such a flexible approach. These gains have been confirmed by means of field trials performed using an SDR implementation of the flexible physical layer. The field trials confirmed the necessity for a flexible approach of the physical layer. Flexibility usage provides the ability to either give superior coverage or alternatively bring larger throughput (particularly for shorter ranges of application). The choice of physical layer modes: Turbo-FSK for longer ranges, OFDM for faster data rates and SC-FDM for fast but lower power applications is confirmed by both simulation results and field trial measurements. Furthermore, field trial comparison with current state of the art solutions such as LoRa proved the gain that the new approach can bring: significant range improvements may be provided with the new approach for similar levels of throughput as supported by current systems.

Further developments should consider the integration of the newly proposed physical layer with a specifically designed MAC approach optimized to select the most appropriate physical layer mode of operation as a function of the transmission conditions and the application requirements.

\section{REFERENCES}

[1] L. Columbus. 2017 Roundup of Internet of Things Forecasts. Forbes, 2018. [Online]. Available: https://www.forbes.com/sites/louiscolumbus/2017/12/10/2017roundup-of-internet-of-things-forecasts/\#66ae9cf71480. [Accessed: Oct2018]

[2] U. Raza, P. Kulkarni, and M. Sooriyabandara, "Low power wide area networks: An overview," IEEE Communications Surveys Tutorials, vol. 19, no. 2, pp. 855-873, Secondquarter 2017.

[3] 3GPP, "Evolved Universal Terrestrial Radio Access (E-UTRA); Physical channels and modulation," 3rd Generation Partnership Project (3GPP), Technical Specification (TS) 36.211, 03 2017, version 14.2.0.

[4] V. Mannoni, V. Berg, F. Dehmas, and D. Noguet, "A flexible physical layer for lpwa applications," in Cognitive Radio Oriented Wireless Networks, P. Marques, A. Radwan, S. Mumtaz, D. Noguet, J. Rodriguez, and M. Gundlach, Eds. Cham: Springer International Publishing, 2018, pp. $322-333$.

[5] Y. Roth, J.-B. Doré, L. Ros, and V. Berg, "Turbo-FSK: A New Uplink Scheme for Low Power Wide Area Networks," in 2015 IEEE 16th International Workshop on Signal Processing Advances in Wireless Communications (SPAWC), Stockholm, Sweden, June 2015, pp. 81-85.

[6] Y. Roth, J.-B. Dore, L. Ros, and V. Berg, "Coplanar Turbo-FSK: A Flexible and Power Efficient Modulation for the Internet-of-Things," Wireless Communications and Mobile Computing, Article ID 3072890, 17 pages,2018. [Online]. Available: https://doi.org/10.1155/2018/3072890

[7] V. Berg, J. Dore, and D. Noguet, "A multiuser FBMC receiver implementation for asynchronous frequency division multiple access," in 17th Euromicro Conference on Digital System Design, DSD 2014 Verona, Italy, August 27-29, 2014, 2014, pp. 16-21. [Online]. Available: https://doi.org/10.1109/DSD.2014.11

[8] "EURO-COST 231: Digital Mobile Radio Towards Future Generation Systems, ," Tech. Rep., European Cooperation in the Field of Scientific and Technical Research, COST231 Final Report. [Online]. Available: http://www.lx.it.pt/cost231/

[9] M. K. Raja, X. Chen, Y. D. Lei, Z. Bin, B. C. Yeung, and Y. Xiaojun, "A $18 \mathrm{mw}$ tx, $22 \mathrm{mw}$ rx transceiver for $2.45 \mathrm{ghz}$ ieee 802.15.4 wpan in 0.18-m cmos," in 2010 IEEE Asian Solid-State Circuits Conference, Nov 2010, pp. 1-4.

[10] S. Cui, A. J. Goldsmith, and A. Bahai, "Energy-constrained modulation optimization," IEEE Transactions on Wireless Communications, vol. 4, no. 5, pp. 2349-2360, Sept 2005.

[11] Y. Roth, J.-B. Doré, L. Ros, and V. Berg, A Comparison of Physical Layers for Low Power Wide Area Networks. Springer International Publishing, 2016. 